\title{
UNIVERSIDADE DE SÃO PAULO FCF/FEA/FSP
}

Programa de Pós-Graduação Interunidades em Nutrição Humana Aplicada

Efeitos da renda sobre atributos da alimentação no Brasil: Uma análise da influência de medidas associadas ao salário mínimo sobre dimensões da segurança alimentar e nutricional

André Bento Chaves Santana

Tese apresentada ao Programa de Pós-Graduação Interunidades em Nutrição Humana Aplicada para obtenção do título de Doutor em Ciências.

Orientadora: Profa. Dra. Flavia Mori Sarti

São Paulo

2018 


\section{RESUMO}

\section{SANTANA, A.B.C. Efeitos da renda sobre atributos da alimentação no Brasil: Uma}

análise da influência de medidas associadas ao salário mínimo sobre dimensões da segurança alimentar e nutricional. 2018. 118f. Tese (Doutorado em Nutrição Humana Aplicada). Faculdade de Ciências Farmacêuticas/Faculdade de Economia, Administração e Contabilidade/Faculdade de Saúde Pública, Universidade de São Paulo (USP), São Paulo, 2018.

O objetivo do presente trabalho foi avaliar adequação do uso do salário mínimo, em comparação com outras medidas de renda, na investigação de aspectos de segurança alimentar e nutricional no Brasil; especialmente quanto à representatividade do poder aquisitivo, à qualidade nutricional do padrão alimentar da população e às alternativas para solução de situações de insegurança alimentar. A partir de dados disponibilizados pelo Instituto Brasileiro de Geografia e Estatística (IBGE) e pelo Departamento Intersindical de Estatística e Estudos Econômicos (DIEESE), foram conduzidas análises sobre: (1) evolução do poder aquisitivo do salário mínimo desde sua criação até atualmente, em comparação ao valor monetário necessário para aquisição dos itens alimentares inicialmente previstos na cesta básica utilizada para seu cálculo; (2) adequação nutricional da cesta básica de alimentos em relação às recomendações nutricionais vigentes na atualidade; (3) qualidade nutricional do consumo alimentar da população brasileira em comparação à dieta baseada em itens da cesta básica pela aplicação do Índice de Qualidade da Dieta Revisado; (4) caracterização de domicílios brasileiros em situação de insegurança alimentar para mapeamento das atitudes adotadas em resposta à escassez de alimentos. Os resultados obtidos indicaram deterioração da relevância do salário mínimo no contexto alimentar e nutricional, devido à ausência de atualizações monetárias de valor em períodos críticos da inflação brasileira e à mudança nas recomendações nutricionais em decorrência da evolução do conhecimento na área, sem correspondente revisão da composição da cesta de alimentos originalmente proposta como base para cálculo do salário mínimo. $\mathrm{O}$ padrão de consumo alimentar da população brasileira e a dieta baseada no consumo dos itens da cesta básica de alimentos foram caracterizados por baixo consumo de frutas, vegetais e cereais, além de excesso de ingestão energética proveniente de gorduras e açúcar. Em relação à insegurança alimentar, observou-se preferência por atitudes de redistribuição intradomiciliar de alimentos ou busca de apoio em rede social proximal dos indivíduos em situação de insegurança alimentar. Programas governamentais raramente são mencionados como parte das ações principais para mitigar problemas de escassez de alimentos no contexto domiciliar. A partir do conjunto de evidências analisadas, conclui-se que políticas de reajuste do salário mínimo e de distribuição de renda apresentam baixa efetividade em termos de promoção de um melhor padrão alimentar para população brasileira. Determinados programas governamentais direcionados à promoção da segurança alimentar e nutricional possivelmente apresentam insuficiência na cobertura ou baixa divulgação entre segmentos populacionais de menor renda, colocando em risco a garantia do acesso à alimentação adequada no Brasil.

Palavras-chaves: salário mínimo; cesta básica de alimentos; segurança alimentar e nutricional; Índice de Qualidade da Dieta; insegurança alimentar; análise de redes. 


\begin{abstract}
SANTANA, A.B.C. Effects of income on food attributes in Brazil: An analysis of the influence of measures associated to the minimum wage on food and nutritional security dimensions. 2018. 118p. Thesis (Doctorate in Applied Human Nutrition). School of Pharmaceutical Sciences/School of Economics, Administration and Accounting/School of Public Health, University of São Paulo (USP), São Paulo, 2018.
\end{abstract}

The objective of the study was to assess the adequacy of the use of minimum wage in the investigation of aspects related to food and nutrition security in Brazil, in comparison to other income measures; especially referring to its characteristics in representing purchase power, nutritional quality of food consumption patterns at population level and alternatives for solution of food insecurity situations. Using data from the Brazilian Institute for Geography and Statistics (IBGE) and the Brazilian Interunion Department for Statistics and Economic Studies (DIEESE), a set of analysis was performed in relation to: (1) evolution of purchase power of the Brazilian minimum wage from its inception until nowadays, in comparison to the monetary value required to acquisition of the food items originally proposed in the basic food basket used for its calculation; (2) nutritional adequacy of the Brazilian basic food basket in comparison to recent nutritional recommendations; (3) nutritional quality of the food consumption patterns from the Brazilian population in comparison to a diet based on items of the basic food basket, using the Healthy Eating Index Revised adapted for Brazil; (4) characterization of Brazilian households under food insecurity in order to map attitudes adopted to deal with food scarcity. The results showed deterioration of the relevance of the Brazilian minimum wage in the food and nutrition context, due to the absence of updates in its monetary value during periods of high inflation in Brazil, and due to changes in nutritional recommendations derived from advances in knowledge without revision of the composition of the basic food basket originally proposed for estimation of the minimum wage. The Brazilian population dietary pattern nowadays and the diet based on the basic food basket were marked by low proportion of fruits, vegetables and cereals; and excess of calories from fats, oils and sugar. In relation to food insecurity, results pointed to preference for adoption of attitudes related to intra-household food redistribution and support of social networks in order to deal with lack of food. Government programs were rarely mentioned as potential solutions for reducing the impacts of food scarcity in the household context. The evidences analyzed in the study indicated that policies for adjustment of the minimum wage and programs for income distribution in Brazil had low effectiveness for promotion of improvements in food consumption patterns among the Brazilian population. Certain government programs directed towards promotion of food security probably present insufficient coverage or low dissemination among low income segments of the population, compromising the assurance of adequate access to food in Brazil.

Keywords: minimum wage; food basket; food and nutrition security; Diet Quality Index Revised; food insecurity; network analysis. 


\section{INTRODUÇÃO}

O salário mínimo representa uma forma de proteção contra exploração dos trabalhadores, embora apresente limitações decorrentes da fixação do piso salarial em valores baixos, assim como devido à eminência do desemprego em determinadas situações (SABOIA, 1985). Desde sua implantação em $1^{\circ}$. de maio de 1940, a partir do Decreto Lei $\mathrm{n}^{\mathrm{o}}$. 2.162, o salário mínimo teve diversos reajustes que marcam períodos de valorização, estagnação e perdas de poder aquisitivo, de acordo com cenário político e econômico brasileiro.

Considerando alimentação como necessidade básica para sobrevivência que compromete significativa parte do orçamento familiar entre indivíduos de baixa renda, instituiu-se o Decreto Lei $n^{\circ}$. 399, de 30 de abril de 1938, que definiu a chamada "ração essencial" do salário mínimo, constituída de doze grupos de alimentos organizados segundo possibilidade de substituição, à exceção do leite (considerado alimento essencial). O teor energético, proteínas, cálcio, ferro e fósforo, denominados "elementos nutritivos", eram principais determinantes da ração essencial (SABOIA, 1985).

A decisão acerca das quantidades de cada alimento que constavam na ração essencial foi realizada pelas Comissões do Salário Mínimo em 1938, a partir de quantidades suficientes dos nutrientes para sustento de um trabalhador adulto em três diferentes regiões do país. Atualmente, a ração essencial é utilizada como base da pesquisa mensal da Cesta Básica Nacional (CBN), realizada pelo Departamento Intersindical de Estatística e Estudos Econômicos (DIEESE), no contexto do monitoramento da evolução mensal de preços dos produtos da cesta básica e estimativa do gasto mensal de um trabalhador para aquisição alimentar (DIEESE, 2016).

A proposição de políticas públicas para garantia das necessidades básicas de alimentação de uma população depende de análises detalhadas de determinados parâmetros, incluindo padrão de consumo alimentar e variáveis sociodemográficas e econômicas. Tais parâmetros devem pautar a elaboração de medidas governamentais efetivas, que busquem assegurar a subsistência e a qualidade de vida de indivíduos e populações (TEIXEIRA, 2002).

Assim, torna-se necessário compreender efeitos do uso de medidas associadas ao salário mínimo na investigação das dimensões da segurança alimentar e nutricional da população. A evolução do poder aquisitivo do salário mínimo como política pública essencial na garantia do direito do cidadão à alimentação é pautada em remuneração adequada do trabalho para promoção de padrões de vida apropriados. 
Salienta-se, adicionalmente, a importância da análise da qualidade nutricional do padrão alimentar da população, especialmente entre grupos populacionais de baixa renda, em comparação à composição da cesta básica de alimentos utilizada como base de cálculo do salário mínimo, no que tange às recomendações nutricionais atualmente vigentes. Por fim, diante de situações de insegurança alimentar e nutricional, torna-se essencial investigar estratégias utilizadas no âmbito domiciliar para enfrentamento do problema de escassez alimentar, segundo perfil sociodemográfico e econômico dos domicílios afetados pela falta de alimentos.

Considerando o papel do salário mínimo no contexto das políticas públicas na área de segurança alimentar e nutricional, as hipóteses analisadas no presente estudo foram: (1) Há inviabilidade de uso do salário mínimo como medida padrão de renda em pesquisas da área de nutrição em saúde pública, tendo em vista ausência de ações para manutenção do poder aquisitivo real; (2) Há significativas diferenças entre custos e valores nutricionais expressos pela cesta de alimentos utilizada como base de cálculo do salário mínimo em relação ao padrão alimentar brasileiro atual e às recomendações nutricionais vigentes; (3) As ações governamentais para atenuar problemas relacionados à escassez de alimentos não são alternativas usuais empregadas como estratégia de combate à insegurança alimentar entre domicílios de baixa renda no Brasil.

A estrutura do trabalho inclui os seguintes tópicos, além da presente introdução: delimitação dos objetivos do estudo; referencial teórico sobre vínculo entre salário mínimo, segurança alimentar e transição nutricional no Brasil; metodologia empregada na investigação das hipóteses apresentadas; resultados do estudo, descritos na forma de quatro manuscritos a serem submetidos para publicação em periódicos; e, por fim, conclusão da tese, a partir da síntese dos resultados obtidos e reflexões acerca dos efeitos diferentes medidas de renda, incluindo salário mínimo, sobre atributos da segurança alimentar e nutricional no Brasil. 


\section{CONCLUSÕES}

Políticas de reajuste do salário mínimo e distribuição de renda são importantes determinantes da segurança alimentar e nutricional entre famílias de baixa renda, tendo em vista possibilidade de acesso aos alimentos pela via econômica. A falta de recursos para custear necessidades básicas com alimentação a partir dos rendimentos familiares constitui sinal de inefetividade ou falta de ação governamental para correção das falhas de mercado, que resultam na quebra da garantia ao direito humano à alimentação adequada.

A defasagem do poder aquisitivo do salário mínimo e do valor nutricional da cesta básica de alimentos frente às necessidades alimentares da população brasileira e às recomendações nutricionais atuais constitui ponto crítico na busca por implementação de políticas públicas para garantia de segurança alimentar e nutricional no país.

O presente estudo apontou inadequações no valor nutricional e na qualidade da dieta representadas pelo padrão alimentar atual da população brasileira e pela cesta básica de alimentos, base do cálculo do salário mínimo no país; assim como deterioração do poder aquisitivo do salário mínimo em vários períodos da história brasileira recente.

Assim, torna-se necessária revisão dos fundamentos de remuneração dos trabalhadores, acompanhada de adequação na composição da cesta básica, especialmente no que tange à promoção de uma alimentação saudável pela complementação da dieta com frutas, vegetais, cereais, leite e derivados. $\mathrm{O}$ incremento do consumo de alimentos processados e ultraprocessados constitui fator preocupante em termos de tendência do padrão alimentar nacional, assim como avaliação de sua qualidade global.

Ademais, o mapeamento de estratégias para enfrentamento do problema da insegurança alimentar ocasionada pela incerteza ou quebra do acesso regular a alimentos demonstram insuficiência dos programas governamentais vigentes voltados para segmentos da população em situação de maior vulnerabilidade socioeconômica.

O conjunto de evidências reunidas no presente trabalho permitiu compreender a influência de fatores relacionados ao salário mínimo e renda no cenário de segurança alimentar e nutricional, que colocam em risco a garantia de acesso à alimentação adequada no Brasil. 


\section{REFERÊNCIAS}

AFONSO, L.E.; PEREDA, P.C.; GIAMBIAGI, F.; FRANCO, S. O salário mínimo como instrumento de combate à pobreza extrema: Estariam esgotados seus efeitos? Economia Aplicada, v. 15, n. 4, p. 559-593, 2011.

ALEXANDRATOS, Nikos et al. World agriculture towards 2030/2050: the 2012 revision. Rome, FAO: ESA Working paper, 2012.

ANDRADE, S.C.; PREVIDELLI, A.N.; MARCHIONI, D.M.; FISBERG, R.M. Avaliação da confiabilidade e validade do Índice de Qualidade da Dieta Revisado. Revista de Saúde Pública, v. 47, n. 4, p. 675-683, 2013.

BARRETTO, Sérgio Augusto Jábali; CYRILLO, Denise Cavallini. Análise da composição dos gastos com alimentação no Município de São Paulo (Brasil) na década de 1990. Rev Saúde Pública, v. 35, n. 1, p. 52-9, 2001.

BECKER, Cláudio; SACCO DOS ANJOS, Flávio. Segurança alimentar e desenvolvimento rural: limites e possibilidades do Programa de Aquisição de Alimentos da agricultura familiar, em municípios do Sul gaúcho. Segurança Alimentar e Nutricional, Campinas, v. 17, n. 1, p. $61-72,2010$.

BELIK, Walter et al. Perspectivas para segurança alimentar e nutricional no Brasil. Saúde e sociedade, 2003.

BOLAND, Mike J. et al. The future supply of animal-derived protein for human consumption. Trends in food science \& technology, v. 29, n. 1, p. 62-73, 2013.

BORGES, Camila Aparecida et al. The cost of meeting dietary guidelines for low-income Brazilian families. Cadernos de Saúde Pública, v. 31, n. 1, p. 137-148, 2015.

BRASIL. Constituição dos Estados Unidos do Brasil, de 18 de setembro de 1946. Rio de Janeiro, 1946.

BRASIL. Constituição da República Federativa do Brasil de 1988. Brasília, 1988. 
BRASIL. Decreto-lei 399, de 30 de abril de 1938. Brasília: Diário Oficial da União, 1938.

BRASIL. Lei n. 185, de 14 de janeiro de 1936. Brasília: Diário Oficial da União, 1936.

BRASIL. Lei n. 11.346 de setembro de 2006. Brasília: Diário Oficial da União, 2006.

BRASIL. Ministério da Saúde. Guia alimentar para a população brasileira. Brasília: Ministério da Saúde, 2008.

BURLANDY, Luciene. Transferência condicionada de renda e segurança alimentar e nutricional. Ciência \& Saúde Coletiva, v. 12, n. 6, p. 1441-1451, 2007.

CAMARGO, José Marcio; NERI, Marcelo; GONZAGA, Gustavo. Salário mínimo, efeito farol e pobreza. Revista de economia política, v. 21, n. 2, p. 82, 2001.

CAMPOS, R.R. Josué de Castro e o direito à alimentação. Geografia em Questão, v. 5, n. 1, p. 28-46, 2012.

CASTRO, J. Alimentação e raça. Vol. 5. Rio de Janeiro: Bibliotheca de Divulgação Scientifica, 1936.

CASTRO, J. Condições de vida das classes operárias do Recife: um estudo econômico de sua alimentação. $1^{\text {a }}$ edição. Recife, 1932.

CASTRO, J. Geografia da fome. $10^{\text {a }}$ edição revista. Rio de Janeiro: Editora Antares, 1952.

CASTRO, J. Salário mínimo. Rio de Janeiro, 1935.

CASTRO, J. O problema da alimentação no Brasil. $3^{\text {a }}$ edição aumentada. São Paulo: Companhia Editora Nacional, 1939.

CERVATO, A.M.; VIEIRA, V.L. Índices dietéticos na avaliação da qualidade global da dieta. Revista de Nutrição, v. 16, n. 3, p.347-355, 2003. 
CERVATO-MANCUSO, Ana Maria; GOMES, Fiore Elaine; DA SILVA, Redolfi Solange Cavalcante. Guia de segurança alimentar e nutricional. Editora Manole, 2015.

CYRILLO, Denise Cavallini; SAES, Maria Sylvia Macchione; BRAGA, Márcio Bobik. Tendências do consumo de alimentos e o Plano Real: uma avaliação para a Grande São Paulo. Planejamento e Políticas Públicas, n. 16, 2009.

CONSEA. A segurança alimentar e nutricional e o direito humano à alimentação adequada no Brasil: Indicadores e monitoramento da Constituição de 1988 aos dias atuais. 2010.

COTTA, Rosângela Minardi Mitre; MACHADO, Juliana Costa. Programa Bolsa Família e segurança alimentar e nutricional no Brasil: revisão crítica da literatura. 2013.

COHEN, E,; FRANCO, R. Avaliação de projetos sociais. 5ª Edição. Petrópolis: Vozes; 2002.

DA SILVA, Carlos Lavalle; DE ALMEIDA, Fernando Galvão. Os efeitos da redução do ICMS na cesta básica. Revista Conjuntura Econômica, v. 48, n. 10, p. 22-23, 1994.

DELGADO, Guilherme C.; DA CONCEIÇÃO, Júnia Cristina PR; OLIVEIRA, Jader José de. Avaliação do programa de aquisição de alimentos da agricultura familiar (PAA). 2005.

DEPARTAMENTO INTERSINDICAL DE ESTATÍSTICA E ESTUDOS ECONÔMICOS (DIEESE). Cesta Básica Nacional - Metodologia. São Paulo, 1993.

DEPARTAMENTO INTERSINDICAL DE ESTATÍSTICA E ESTUDOS ECONÔMICOS (DIEESE). Cesta Básica Nacional - Metodologia. São Paulo, 2016.

DEPARTAMENTO INTERSINDICAL DE ESTATÍSTICA E ESTUDOS ECONÔMICOS (DIEESE). Salário mínimo: Instrumento de combate à desigualdade. São Paulo: DIEESE, 2010.

EMBRAPA. Sustentabilidade e sustentação da produção de alimentos no Brasil: O papel do País no cenário global. - Brasília: Centro de Gestão e Estudos Estratégicos, 2014. 
EVENSON, Robert E.; GOLLIN, Douglas. Assessing the impact of the Green Revolution, 1960 to 2000 . Science, v. 300, n. 5620, p. 758-762, 2003.

FISBERG, R.M.; VILLAR, B.S. Manual de receitas e medidas caseiras para cálculo de inquéritos alimentares. 1a.Edição, São Paulo, Ed.Signus, 2002.

FOOD AND AGRICULTURE ORGANIZATION. The State of Food Insecurity in the World 2001. Rome, 2002.

FOOD AND AGRICULTURE ORGANIZATION. Human energy requirements: Report of a Joint FAO/WHO/UNU Expert Consultation. 2004.

FREITAS, Leandro Quintanilha. Medidas de centralidade em grafos. 2010. Tese de Doutorado. dissertação de mestrado, Universidade Federal do Rio de Janeiro.

GIAMBIAGI, F.; FRANCO, S. O esgotamento do papel do salário mínimo como mecanisalário mínimoo de combate à pobreza extrema. Texto para Discussão 1290. Rio de Janeiro: IPEA, 2007.

GUANZIROLI, Carlos Enrique. Agricultura familiar e reforma agrária no século XXI. Editora Garamond, 2001, p. 15-22.

HAINES, P.S.; SIEGA-RIZ, A.M.; POPKIN, B.M. The Diet Quality Index Revised: A measurement instrument for populations. Journal of the American Dietetic Association, v. 99, p. 697-704, 1999.

INSTITUTO BRASILEIRO DE GEOGRAFIA E ESTATÍSTICA (IBGE). Pesquisa de Orçamentos Familiares 2008-2009. Rio de Janeiro: FIBGE, 2009a.

INSTITUTO BRASILEIRO DE GEOGRAFIA E ESTATÍSTICA (IBGE). Pesquisa de Orçamentos Familiares 2008-2009. Tabela de composição nutricional dos alimentos consumidos no Brasil. Rio de Janeiro: FIBGE, 2009b.

KEPPLE, Anne Walleser; SEGALL-CORRÊA, Ana Maria. Conceituando e medindo segurança alimentar e nutricional. Ciência \& Saúde Coletiva, v. 16, p. 187-199, 2011. 
LAVINAS, Lena. Acessibilidade alimentar e estabilização econômica no Brasil nos anos 90. Rio de Janeiro: IPEA, 1998.

LEVY-COSTA, Renata Bertazzi et al. Disponibilidade domiciliar de alimentos no Brasil: distribuição e evolução (1974-2003). Revista de Saúde Pública, v. 39, n. 4, p. 530-540, 2005.

LEVY, Renata Bertazzi et al. Regional and socioeconomic distribution of household food availability in Brazil, in 2008-2009. Revista de Saúde Pública, v. 46, n. 1, p. 06-15, 2012.

LOUZADA, Maria Laura da Costa et al. Ultra-processed foods and the nutritional dietary profile in Brazil. Revista de saude publica, v. 49, p. 00-00, 2015.

MACEDO, R; GARCIA, M.E. Observações sobre a política brasileira de salário mínimo. São Paulo: Fundação Instituto de Pesquisas Econômicas, 1978.

MALUF, Renato S.; SPERANZA, Juliana. Volatilidade dos preços internacionais e inflação de alimentos no Brasil: fatores determinantes e repercussões na segurança alimentar e nutricional. 2013.

MATTOSO, J.E.L. O mínimo salário mínimo. Revista São Paulo em Perspectiva, v. 2, ano 3, p. 26-36, 1988.

NATIONAL ACADEMY OF SCIENCES. Dietary Reference Intakes. Food and Nutrition Board, Institute of Medicine, National Academies, 2004.

NODARI, Rubens Onofre; GUERRA, Miguel Pedro. A agroecologia: estratégias de pesquisa e valores. Estudos Avançados, v. 29, n. 83, p. 183-207, 2015.

NÚCLEO DE ESTUDOS E PESQUISAS EM ALIMENTAÇÃO (NEPA). Tabela Brasileira de Composição de Alimentos. 4ª Edição. Campinas: NEPA/UNICAMP, 2011.

PARFITT, Julian; BARTHEL, Mark; MACNAUGHTON, Sarah. Food waste within food supply chains: quantification and potential for change to 2050.Philosophical Transactions of the Royal Society of London B: Biological Sciences, v. 365, n. 1554, p. 3065-3081, 2010 
PREVIDELLI, A.N.; ANDRADE, S.C.; FERREIRA, S.R.G.; FISBERG, R.M.; MARCHIONI, D.M. Índice de Qualidade da Dieta Revisado para população brasileira. Revista de Saúde Pública, v. 45, n. 4, p. 794-798, 2011.

ROSSI, P.H.; LIPSEY, M.W.; FREEMAN, H.E. Evaluation: A systematic approach. $7^{\text {th }}$. edition. London: Sage Publications; 2004.

SABOIA, João. Salário mínimo no Brasil: a experiência brasileira. L\&PM Editores, 1985.

SABOIA, J. Efeitos do salário mínimo sobre a distribuição de renda no Brasil no período 1995/2005: Resultados de simulações. Econômica, v. 9, n. 2, p. 270-295, 2007.

SANTILLI, Adimir Umberto Valentim Raga; DE OLIVEIRA, Larissa Yamazaki. Salário Mínimo Brasileiro: uma análise constitucional. 2015.

SARAIVA, Elisa Braga et al. Panorama da compra de alimentos da agricultura familiar para o Programa Nacional de Alimentação Escolar. Cien Saude Colet, v. 18, n. 4, p. 927-936, 2013.

SARTI, F.M. Dos microindicadores à macropolítica: Uma proposta de avaliação econômica em políticas públicas de alimentação e nutrição. Tese (Livre Docência) - Escola de Artes, Ciências e Humanidades da Universidade de São Paulo, 2010.

SOUZA, P.R; BALTAR, P.E. Salário mínimo e a taxa de salários no Brasil. Pesquisa e Planejamento Econômico, v. 9, n. 3, p. 629-659, 1979.

TEIXEIRA, Elenaldo Celso. O papel das políticas públicas no desenvolvimento local e na transformação da realidade. Salvador: AATR, 2002.

UNITED STATES DEPARTMENT OF AGRICULTURE (USDA). The Healthy Eating Index. Washington, DC: USDA; 1995.

UNIVERSIDADE DE SÃO PAULO / FOOD RESEARCH CENTER (USP/FORC). Tabela Brasileira de Composição de Alimentos (TBCA). Versão 6.0. São Paulo, 2017. [Acesso em: 20 de junho de 2018]. Disponível em: http://www.fcf.usp.br/tbca/ 
VARIYAM, J.N.; BLAYLOCK, J.; SALÁRIO MÍNIMOALLWOOD, D.; BASIOTIS, P.P. USDA's Healthy Eating Index and nutrition information. Technical Bulletin nr. 1866. Washington, DC: USDA; 1998.

WENDPAP, Loiva Lide et al. Qualidade da dieta de adolescentes e fatores associados. Cadernos de Saúde Pública, v. 30, p. 97-106, 2014. 